\title{
APPLYING WEB AND INFORMATION INTEGRATION TECHNOLOGIES FOR INTENSIFIED CONSTRUCTION PROCESS CONTROL
}

*E. Viljamaa ${ }^{1}$, I. Peltomaa ${ }^{2}$ and T. Seppälä ${ }^{1}$

${ }^{I}$ VTT Technical Research Centre of Finland P.O.Box 1100 FI-90571 Oulu, Finland

(*Corresponding author: esa.viljamaa@vtt.fi)

${ }^{2}$ VTT Technical Research Centre of Finland P.O.Box 3

FI-92101 Raahe, Finland 


\title{
APPLYING WEB AND INFORMATION INTEGRATION TECHNOLOGIES FOR INTENSIFIED CONSTRUCTION PROCESS CONTROL
}

\begin{abstract}
In this research, earlier developed information integration method and novel web technologies are applied to intensify infrastructure construction process control. The applied method uses semantic methods to integrate scattered information that is later on exploited by process management using state-of-the-art web technologies. The applied method and web technologies not only improve the process management situation awareness by integrated up-todate multi-source process data but also provide platform independent access and visualization tool for process data. Utilization of such technologies potentially lead on cost savings, make processes safer and also more environmentally friendly. The integration method also enables intelligent queries to the integrated data making possible to use machine based inferring to the data.
\end{abstract}

\section{KEYWORDS}

Infrastructure building, information integration, semantic methods, web technologies

\section{INTRODUCTION}

Large road and other infrastructure projects are usually very complex and expensive. The project usually consist of tens of parallel and consecutive sub-tasks and project parameters that are affecting to each other and make the project ever-changing monster that is very challenging to manage and to guide to the right direction. Managing and keeping the project on the right track is very challenging task even to the most skilled management personnel. The legislation of safety and eco-efficiency matters and hardening global competition even adds up pressure experienced by project management teams. Above mentioned requirements for the project management is the reason why advanced project management tools are highly valued and needed.

Technological advances have brought new tools for project management and follow-up. Novel positioning, measurement and machine control systems provide process parameter data that earlier were only dreamt of. Project managers may nowadays follow e.g. an excavation subprocess using the data provided by machine control systems. Also the positions of different production units may be known using the GPS systems, etc. There are system providers that may provide an automated machine control and process follow-up for entire sub-process for i.e. road construction purposes that records and visualizes many important process parameters to be used by project management. An asphalt laying process is one good example. There are multiple different control systems from different providers available for the asphalt laying. The problem however remains. There is no control system or method available that could integrate several heterogeneous sub-systems from different vendors.

The infrastructure building topics as well as system integration are vastly researched areas. Kosovac (2007) proposes a framework for information use and management in AEC/FM 
(architecture, engineering, construction and facilities management). El-Diraby et al. (2005) presents domain taxonomy for construction, where the major ontological model is processoriented. Domain ontology was a part of web based knowledge management software, which connected various systems using Web Service technology. (El-Diraby et al., 2005) Isaac and Navon (2012) come to a conclusion that both manually and automatically collected data are required in project monitoring and control and introduce a model which integrates manually and automatically collected data.

Enhancing system integration with semantics and semantic technologies enables more effective information sharing and communication leading to semantic interoperability. Semantic interoperability emphasizes the importance of the information and focuses on enabling content, data, and information to interoperate with software systems outside their origin (Pollock \& Hodgson, 2004). In semantic interoperability the meaning of data is added in the information in a way that it is understandable to both humans and machines. Ontology, which is the prerequisite for building a semantic-based infrastructure, captures consensual knowledge in a generic way to be reused and shared across software applications and by groups of people (Gruber, 1995). Semantic interoperability can be ensured by providing contextual knowledge of domain applications (Ram \& Park, 2004).

In earlier research, required general purpose data integration and control method was introduced. The method uses semantic web technologies and ontologies for general purpose data integration and process control. (Viljamaa et al., 2012) A purpose of the prototype introduced in this paper is to verify the functionality and feasibility of the earlier introduced data integration and control method. The implemented prototype system is called Dynamic Site Control Center (DSCC), which main purpose is to enhance the information acquisition during road construction process for the purposes of construction work management. DSCC integrates, refines and visualizes process information acquired from different road construction process participants. The target is to intensify the information management and communication between construction process participants especially during actual construction process, resulting improvements in process management, scheduling, reacting to changes, quality control and construction site safety.

\section{DYNAMIC SITE CONTROL CENTER}

The prototype implementation of DSCC concentrates on the most substantial issues in the road construction from the construction side management point of view. The essential tasks are mass transportation and cutting and creating layers. Correspondingly the most important resources are work machines like excavators and graders and transport equipment. The main software components of DSCC are presented in Figure 1. The data to the DSCC is received from contractors taking part for the construction process. The data may cover e.g. project plans, mass types and amounts, and machine activity times. The data is stored by the contractors to their own systems, so the information formats vary a lot. Therefore data adapter component is needed to collect information from companies' systems and to store the information as a common format to the DSCC server. Server environment component includes inference module, database module and web servers. The information from DSCC is accessible through data exploiter and provider component which contains graphical user interfaces (GUIs). Using GUIs it is also possible to input data to the DSCC, if the data is not received from companies' systems or it is desired to feed manually. 


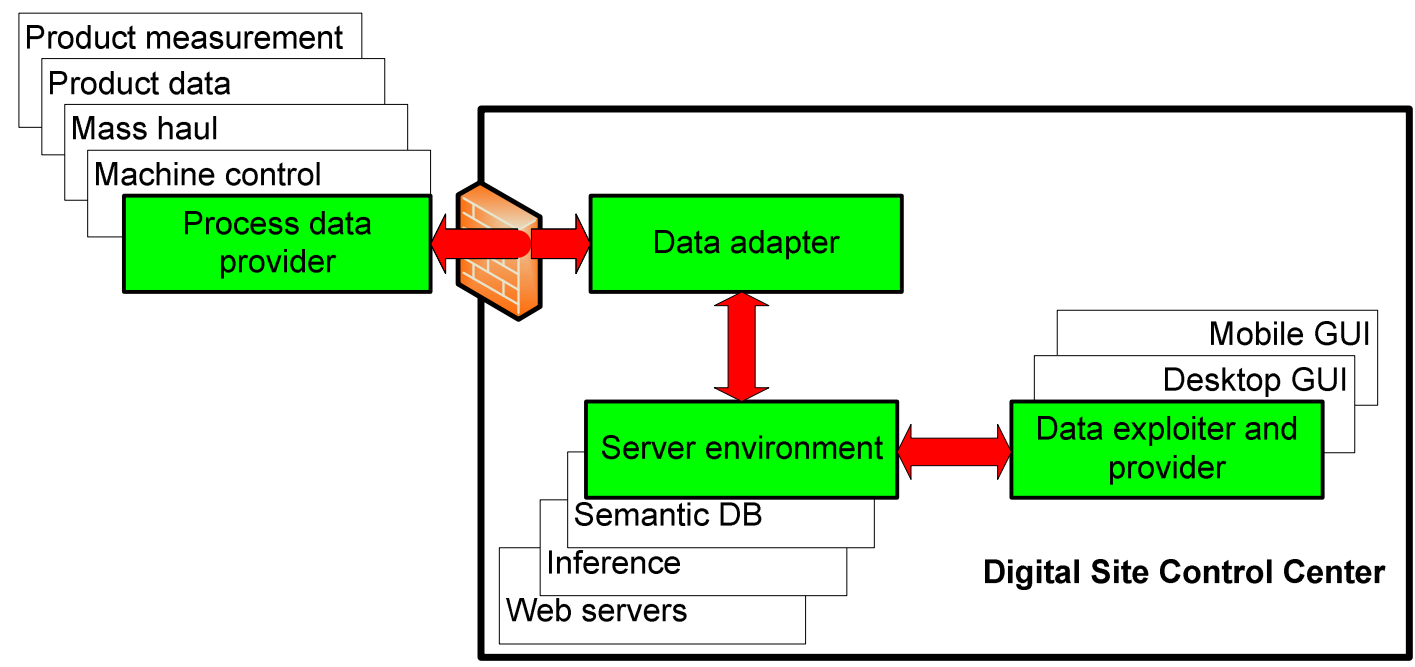

Figure 1 - DSCC software architecture

Inference module does some automated decision making, and it is responsible for combining received data to a one whole and inferencing e.g. it calculates work progress based on activity information from transport equipment and work machines. Database module stores the data combined from data sources that inference module produces, and data that users provide through GUIs, like user information. As database triplestore is used, this enables storing data semantics. Web servers provide DSCC information to data exploiter and provider module to be viewed for users through web GUIs.

\section{Information Flows}

Information flows between data providers, DSCC server environment (DSCC SE) and data exploiters are presented in Figure 2. Data providers and information flows to DSCC SE are presented at left and data exploiters and their information flows are presented at right. Road design information include work plan, line geometry of the road, mass planning, and control models. Road design information includes a list of poles and their locations in real 3D format e.g. LandXML derivative InfraModel2. (Inframodel2, 2013) Mass amounts are defined for certain pole in certain layer of the road. In our case the mass information from planning software is provided as ASCII files. Machine control models for each layer are provided in DWG, DXF or other form for visualisation. Production plans include mass transport and excavation plans, project tasks, and schedules. DSCC SE supports scheduling and tasking which is done using Microsoft Project software or other software supporting similar format. Diagnostics and measurement companies produce laser point clouds which can be used for layer examination or road diagnostics. Work machine information includes work machine status, activity, work time, and location. Mass transportation information contains information about transport equipment's location and status, transportation status, and load's mass amount and type. 


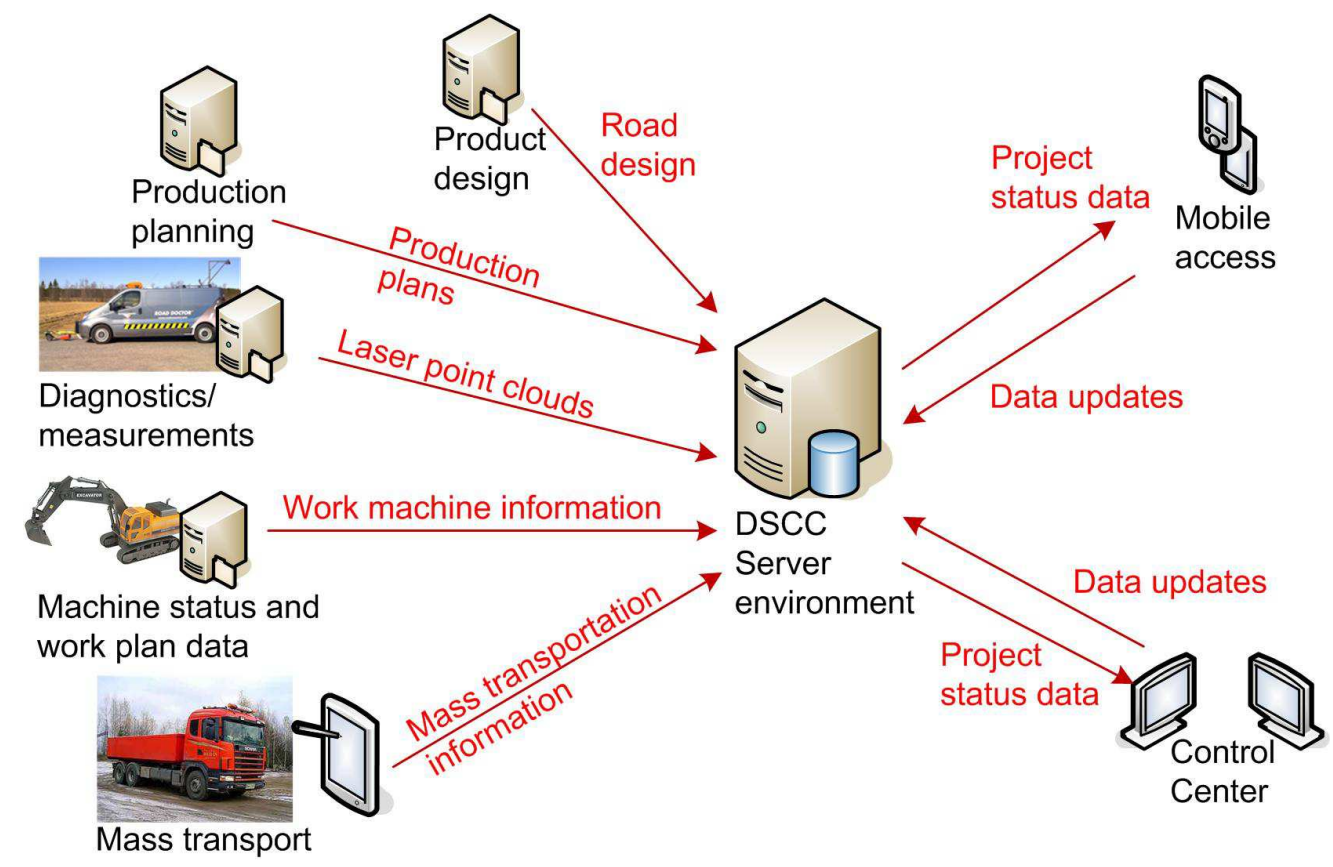

Figure 2 - Information flows

For data exploiters DSCC offers web-based status and plan view of project and tasks, human resources and vehicles of the project in graphical (maps, images) and textual manners. The views rely on a mobile device optimized GUI API resulting easy access from an office PC as well as a mobile device in the field.

\section{Dynamic User Interface and Toolchain}

The DSCC GUIs are implemented by using up-to-date web technologies which enable device and application independent development and use.

Figure 3Figure 3 presents the map view of the DSCC.

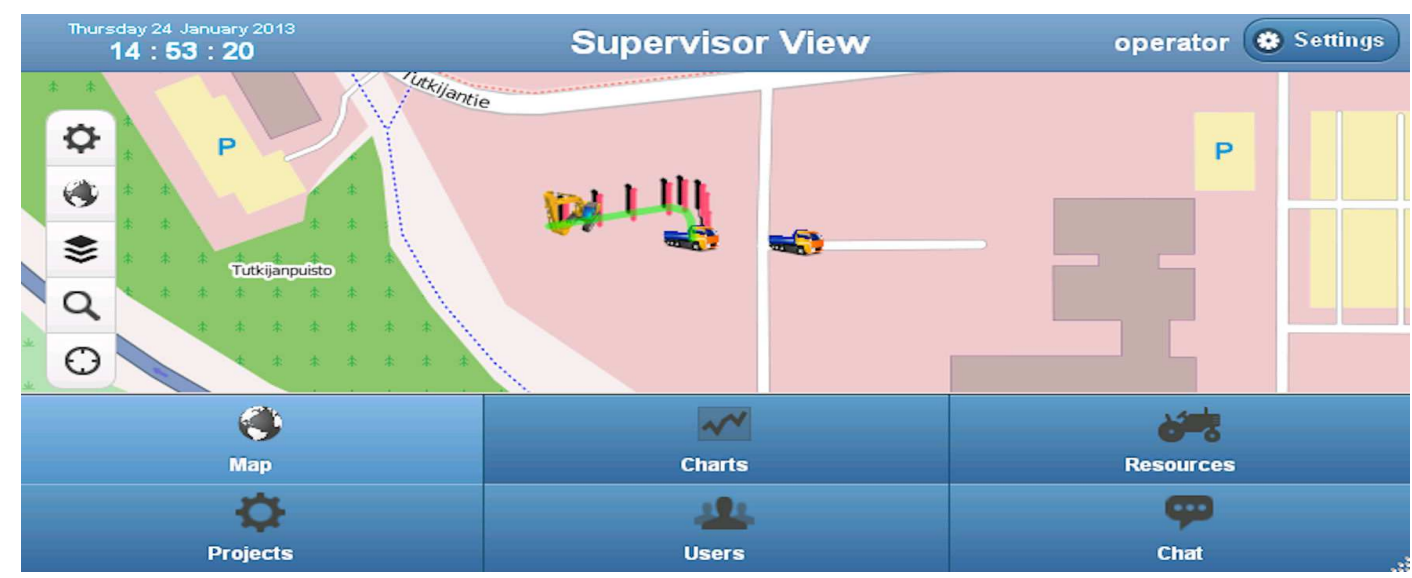

Figure 3 - DSCC main view 


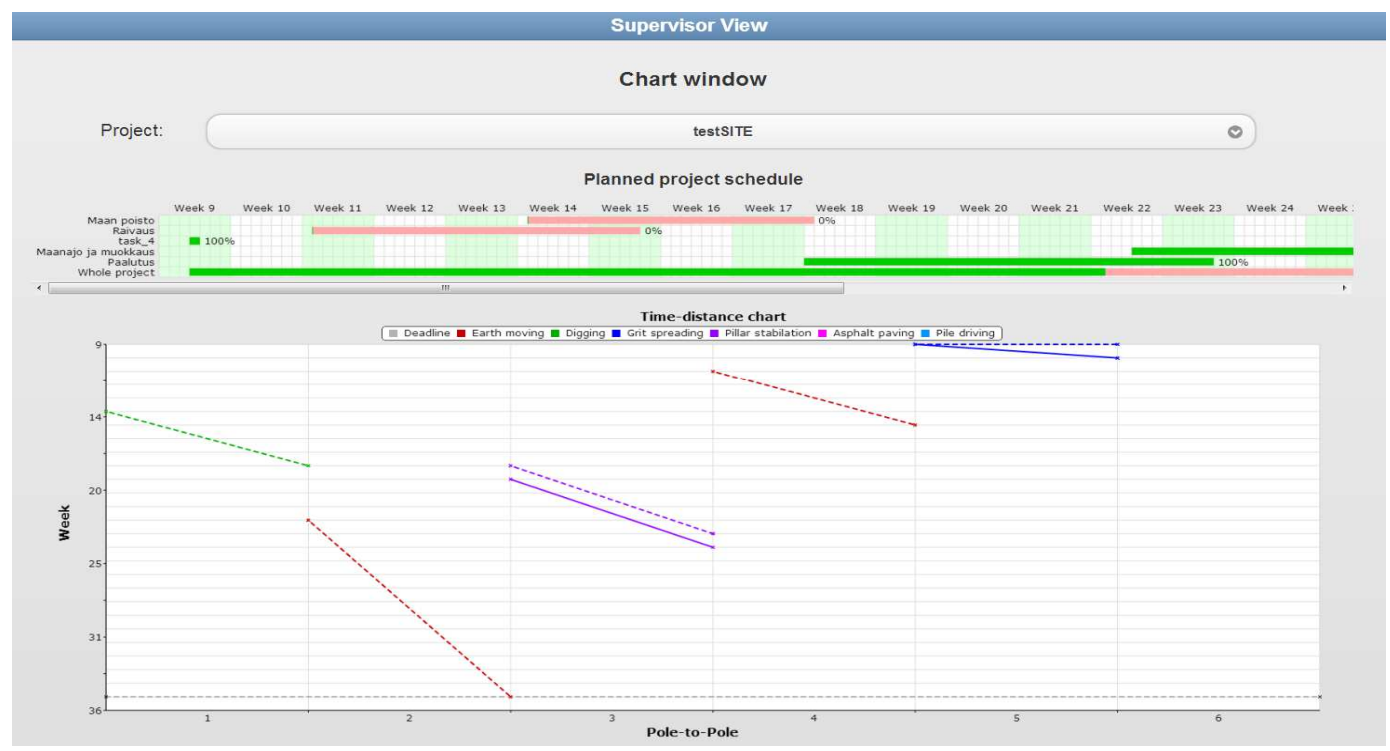

Figure 4 - Gantt chart and flow-line chart of a project

The project management staff can view and alter different process data and messaging properties using 6 different sub-views. These views contain data of projects, tasks, locations, users, resources and process status. An example sub-view of Gantt and flow-line charts can be seen in Figure 4.

The toolchain used in implementation of DSCC is presented in Figure 5Figure 5. The implementation was done with three programming languages, and as database query language SPARQL was used. The ontology was created using Protégé framework and OWL Lite language. For querying and storing of data, Sesame framework with OWLIM semantic repository was used. For querying and updating the information from Sesame triplestore, SPARQL 1.1 query language was used. To enable communication with Java and PHP codes, PHP/Java Bridge with Tomcat as servlet engine was used. Dynamic web pages were implemented using combination of PHP and JQuery JavaScript library.

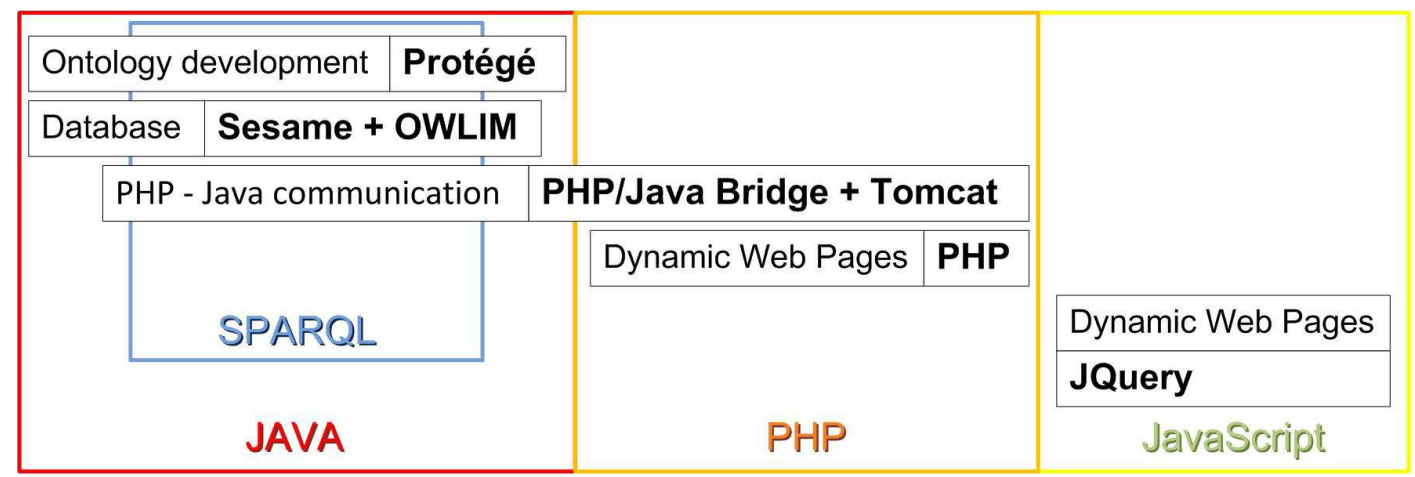

Figure 5 - DSCC toolchain 


\section{RESULTS}

For implemented DSCC prototype some requirements regarding usability and performance were set. The DSCC prototype had to be platform independent, so that it could be used with any common devices and application. The prototype was required to be used through both desktop equipment and mobile devices. Mobility set requirements for e.g. response times, calculation capacity and user interfaces. The prototype should offer information in soft real-time, meaning that in the information acquiring and updating the delay is calculated in tens of seconds. The response time of the prototype should be suitable for web browsing, so the information should reach the user in seconds. And first of all the basic functionality of the prototype should be met before testing could be started.

Testing procedure of the DSCC was multi-staged. During the implementation phase of each software module they were tested regularly. When all the components were coded, the integration test for components was performed in the lab environment. Real outdoor system testing started with the lightweight tests using personnel cars to emulate trucks and excavators. The final full scale tests are planned to be conducted during spring 2013 in the real worksite.

The implemented system was also tested in different platforms since it was supposed to be platform independent. As a result of the test, it was noticed that among the desktop web browsers there were not that many differences. Different smart phone and tablet browsers comparison pointed out slight differences e.g. in the support of HTML5 caching, geolocation API and overall support for used GUI library JQuery. Differences were shown e.g. in different location accuracies, GUI component rendering orders and GUI transitions.

System real-time requirements were met in the level that is needed in construction process follow-up. However the semantic database performance was time to time affecting to the GUI usability. For example the creation of a new road construction project to the DSCC system took around 20 seconds, but it is tolerable as the project creation done quite seldom. Project creation is a complex process including several different phases which each include massive information loadings to the semantic database. In information retrieving tasks DSCC prototype manages quite well, but there is still margin to improve performance.

\section{DISCUSSION}

In this research, earlier introduced semantic technology based data integration and control method was implemented and applied with the mobile web technologies to provide graphical web user interface based tool for intensified construction process control. This kind of combination may potentially bring drastically improved process control when applied. When process management personnel is able to follow most important process parameters in real-time anywhere by mobile devices, they are potentially way better informed of the process status and therefore quicker to react to the changes in the field. That may bring huge cost-savings, ecoefficiency and improved safety figures.

The applied data integration and control method makes it possible to include any kind of data sources to the information chain. Pre-defined ontologies may be increased by additional subontologies. Added information semantics and inter-relationships enables smart data searches and application of the method also for different purposes other than in-action process management. 
The integration method however, requires common agreement of the data formats between the different counterparts. It also requires quite skilled personnel among the method implementing staff, since the methods enabling semantic technologies are quite new and not mainstream. For the same reason, the toolchain and the database system components are not as mature as it could be hoped.

Exploitation of the method by implementing it and using graphical user interfaces based on standard web technologies was quite straightforward, but arduous job. Most of the technologies used were well-tested. The real problem was caused by non-mature semantic database interfacing and storing components. These components were nor well documented neither stabile and caused problems that consumed surprisingly much research resources. Also the semantic database access was occasionally quite slow. This could be improved by streamlining SPARQL queries and by modifying the set of queries produced by GUI. Overall, the implemented system was working as expected. All the functionality with manual and automated sub-process follow-up was operating well. Only real trouble was sometimes quite poor performance of the semantic database connection.

This research proves that earlier developed data integration and control method can be implemented and used for the infrastructure construction process follow-up. The method also brings potentially huge possibilities for process management and other actions intensification. It also brings possibility to re-use collected process data to other actions than actual process control e.g. for project procurement and cost calculations. However, there are several topics that should be further developed. Firstly, the methods how to effectively agree formats between counterparts are needed. Secondly, the most efficient toolchain for developing and implementing ontologies must be found. Thirdly, the most efficient and scalable database and interfacing technology options need to be determined. And fourthly, the initial push to really deploy this kind of method needs to be found; e.g. the clear earnings principle must be found and proved.

\section{ACKNOWLEDGEMENTS}

This work was conducted in "Control of infrastructure construction process using dynamic co-operated network" - project funded by TEKES (Finnish Funding Agency for Technology and Innovation). The contribution and the feedback by the partaking companies are also highly appreciated.

\section{REFERENCES}

El-Diraby, T. A., Lima, C., \& Feis, B. (2005). Domain Taxonomy for Construction Concepts: Toward a Formal Ontology for Construction Knowledge. Journal of Computing in Civil Engineering, 19(4), 394-406. doi: http://dx.doi.org/10.1061/(ASCE)08873801(2005) 19:4(394)

Gruber, T. R. (1995). Towards principles for the design of ontology used for knowledge sharing. International Journal of Human-Computer Studies, 43(5/6), 907-928. doi: http://dx.doi.org/10.1006/ijhc.1995.1081

Inframodel2 web site, visited 24.1.2013 - http://cic.vtt.fi/projects/inframodel2

Isaac, S., \& Navon, R. (2012). Combining automatically and manually collected data for project monitoring and control. Proceedings of the $29^{\text {th }}$ ISARC. doi: http://dx.doi.org/10.4017/gt.2012.11.02.202.676Kosovac, B. (2007). A Framework for Managing Information from Heterogeneous, Distributed, and Autonomous Sources in the 
Architecture, Engineering Construction, and Facilities Management Domain ( $\mathrm{PhD}$ Thesis). Retrieved from The University of British Columbia, UBC Library website: https://circle.ubc.ca/handle/2429/30899

Park, J., \& Ram, S. (2004). Information systems interoperability: What lies beneath? ACM Transactions on Information Systems, 22(4), 595-632. doi: http://dx.doi.org/10.1145/1028099.1028103

Pollock, J. T., \& Hodgson, R. (2004). Adaptive information. Improving business through semantic interoperability, grid computing, and enterprise integration. Hoboke, NJ: John Wiley \& Sons.

Viljamaa, E., Peltomaa, I., Hovila, J., \& Heikkilä, R. (2012). A general information integration method for improved infrastructure building processes. Proceedings of the $29^{\text {th }}$ ISARC. doi: http://dx.doi.org/10.4017/gt.2012.11.02.229.632 